\title{
Rasch analysis of the University of Washington Self-Efficacy Scale short-form (UW-SES-6) in people with long-standing spinal cord injury
}

\author{
Marcel W. M. Post ${ }^{1,2} \cdot$ Jacinthe J. E. Adriaansen ${ }^{1} \cdot$ Claudio Peter $^{3,4}$ \\ Received: 30 September 2017 / Accepted: 10 May 2018 / Published online: 12 June 2018 \\ (c) International Spinal Cord Society 2018
}

\begin{abstract}
Study design Cross-sectional psychometric study.

Objectives The University of Washington Self-Efficacy Scale (UW-SES) is a measure of self-efficacy regarding managing challenges related to multiple sclerosis or spinal cord injury (SCI) that can be used across disabling conditions. The objective of this study was to examine the psychometric properties of its short form, the UW-SES-6, using the Rasch model.

Setting Community, The Netherlands.

Methods Secondary analysis of data from the ALLRISC study. Participants were 261 individuals with a time since onset of SCI (TSI) for at least 10 years, 18-35 at the onset of SCI, and used a wheelchair in everyday life. Rasch analyses were conducted to examine stochastic ordering (fit), unidimensionality, local dependency, reliability, response scale structure, targeting, and item bias.

Results Median age was 47.8 years (Inter-Quartile Range (IQR) 41.9-55), median TSI was 22 years (IQR 16.8-30.3), $73.6 \%$ were male, $90.4 \%$ had a traumatic SCI, $39.8 \%$ had tetraplegia, and $81.6 \%$ had motor complete SCI. After merging the middle three response categories of item 4, the UW-SES-6 showed satisfactory item fit without local dependence. The PSI was high (0.87). Comparison of the person and item threshold distributions showed satisfactory targeting of the UW-SES- 6 to the study group. No differential item functioning was seen with respect to sex, age, level of education, level and completeness of lesion, and TSI.

Conclusions This study showed the UW-SES-6 to be a scale with sound psychometric properties that can be used as a quick and easy self-report measure of self-efficacy in people with SCI.
\end{abstract}

\section{Introduction}

Spinal cord injury (SCI) may result in paralysis, loss of sensation, and a variety of secondary health conditions

Marcel W. M. Post

m.post@dehoogstraat.nl

1 Center of Excellence for Rehabilitation Medicine, Brain Center Rudolf Magnus, University Medical Center Utrecht and De Hoogstraat Rehabilitation, Utrecht, The Netherlands

2 University of Groningen, University Medical Center Groningen, Department of Rehabilitation Medicine, Groningen, The Netherlands

3 Swiss Paraplegic Research (SPF), Nottwil, Switzerland

4 Department of Health Sciences and Health Policy, University of Lucerne, Lucerne, Switzerland
[1-4]. Further, living with SCI has been associated with lower mental health and life satisfaction [5]. Research has shown psychological characteristics of people with SCI to be important determinants of their quality of life [6, 7]. A core psychological characteristic in adjustment to SCI is selfefficacy (SE). It is defined as one's belief or sense of confidence in his/her own ability to perform a particular task or behavior in the future [8]. SE is considered a beneficial psychological resource since a sense of control facilitates the initiation of, and persistence, in adaptive behavior. A recent systematic review showed high SE to be consistently associated with less anxiety and depression, and greater psychological well-being and life satisfaction in people with SCI [9].

This review also showed that SE was conceptualized and measured in different ways in SCI studies [9]; (1) general SE, or one's belief to cope with a variety of difficult commands in life irrespective of type or context of the issue [10]; (2) disability management SE (DMSE) as the belief in 
one's ability to manage the consequences of their condition in everyday life [11, 12]; and (3) SE with respect to specific activities, such as wheelchair-driving [13] and prevention of pressure sores [14]. The concept of DMSE appears to be a core concept to study adjustment to SCI because it is more specific to the circumstances of living with a disability compared to general SE, but is more general compared to $\mathrm{SE}$ with respect to isolated functional tasks [15].

Several measures of DMSE are available, of which the Moorong Self-Efficacy Scale (MSES) and the University of Washington Self-Efficacy Scale (UW-SES) showed validity for use in people with SCI [12, 16-19]. Of the two, the UWSES seemed particularly attractive because it is a more homogeneous scale. It also has a short-form with only six items, the UW-SES-6, making it easy to incorporate it as a measure of DMSE in a larger measurement battery.

The UW-SES was developed to measure self-perceived confidence in managing challenges related to multiple sclerosis or SCI that could be used across several disabling conditions [12]. It consists of 17 items, which proved to be a unidimensional hierarchical scale in 2-parameter Item-Response Theory (IRT) analyses [12]. Concurrent validity of the UW-SES was shown by a very strong Pearson correlation coefficient (0.83) with Lorig's Chronic Disease Self-Efficacy Scale [11, 12]. Looking for a short-form balancing brevity and precision, Amtmann et al. selected six items, further called the UW-SES-6, that together explained $95 \%$ of the variance of the long version [12]. The UW-SES-6 also showed measurement invariance across four diagnostic groups: SCI, multiple sclerosis, muscular dystrophy, and post-polio syndrome indicating that the items have the same meaning across these diagnostic groups [18].

We translated the UW-SES into Dutch in 2012 (details provided in the Methods section). In a previous publication we reported, like Amtmann et al. [12], that UW-SES-6 scores were independent of demographic and SCI characteristics and were moderately to strongly related to measures of participation, mental health, and life satisfaction [19]. Cronbach's $\alpha$ of the Dutch UW-SES-6 was high (0.90) and principal components analysis showed only one component with an Eigenvalue exceeding 1 (3.96) and explaining $66 \%$ of the variance [19].

Although these results support the unidimensionality and validity of the Dutch UW-SES-6, it is unclear whether it also satisfies the more strict assumptions of the Rasch model. If these assumptions are met, transformation of scores into an interval-level scale is allowed, enabling the study of change in clinical and research settings [20]. Therefore, the goal of the current study was to add to our knowledge on the validity of the UW-SES for measuring DMSE among people with SCI by examining the measurement properties of the Dutch UW-SES-6 using the Rasch model and data from a homogeneous group of people with SCI. We hypothesized that the ordering of responses, unidimensionality, and item hierarchy of the UW-SES-6 would satisfy the respective Rasch criteria, as detailed below.

\section{Methods}

\section{Participants}

A secondary analysis was performed of data from the ALLRISC (Active LifestyLe Rehabilitation Interventions in aging Spinal Cord injury) cross-sectional study [21]. Individuals living with SCI for at least 10 years were included in this study, who were between 18 and 35 years of age at the time of onset of their condition, between 28 and 65 years of age at the time of inclusion, and who used a wheelchair, at least for longer distances $(>500 \mathrm{~m})$. Individuals with insufficient mastery of the Dutch language to respond to an oral interview or to understand test instructions were excluded. Data were collected between November 2011 and February 2014.

\section{Procedure}

Potential participants were recruited through all eight rehabilitation centers with a specialty in SCI rehabilitation in the Netherlands. They were asked to complete a selfreport questionnaire, including the UW-SES-6, 2 weeks before visiting the center for a comprehensive medical check-up, physical tests, and an oral interview by a research assistant. The research protocol was approved by the Medical Ethics Committee of the University Medical Center Utrecht. All participants gave written informed consent.

\section{Instruments}

The UW-SES-6 measures the participants' SE regarding the handling of potential negative consequences of their SCI, what we called DMSE [12]. All items are scored on a 5point scale (1: not at all; up to 5: completely). The range of the total score is 6 (low SE) up to 30 (high SE). This total score can be converted in a $T$-score with an average of 50 and standard deviation of 10 [12]. With permission of the authors, we translated the UW-SES-6 into Dutch. Two experts (one rehabilitation physician, one psychologist) independently translated the items first, compared the results, and negotiated a consensus translation.

Demographic variables included: age at the time of the study, sex, relation (single versus married or stable relationship), nationality (Dutch versus other), having children, level of education (high: college, university), and employment status.

SCI characteristics were assessed by the physician according to the International Standards for Neurological Classification of SCI [22]. American Spinal Injury 
Association Impairment Scale (AIS) grades A and B were categorized as motor complete lesions and AIS grades $\mathrm{C}$ and $\mathrm{D}$ as motor incomplete lesions.

\section{Statistical analyses}

Rasch analyses adopting the Partial Credit Model (PCM) were conducted with RUMM2030 software [23]. Stochastic ordering (fit), unidimensionality, local dependency, reliability, response scale structure, targeting, and item bias were investigated. An introduction to Rasch terminology is provided elsewhere [24].

To examine stochastic ordering, we compared the observed responses to the values predicted by the Rasch model. Item fit is indicated by standardized residuals $(Z$ values) and Chi-square test results, with significant Chisquare tests or $Z$ values exceeding \pm 2.5 indicating misfit [25].

To examine unidimensionality, we grouped items showing a positive or negative loading with the first residual principal component analysis factor and compared these two subgroups for each participant using independent $t$ tests. Unidimensionality is demonstrated if the lower bound of the $95 \%$ confidence interval of the proportion of significant $t$-tests is below 5\% [26].

To examine local dependency, we computed residual correlations between all item pairs. A correlation value higher than 0.2 between a pair of item suggests local dependency, or lack of independence of residuals [27].

Further, we examined the reliability by calculation of the person separation index (PSI). This statistic is analogous to Cronbach's alpha, with 0.70 and above representing acceptable reliability for group comparisons [28].

Also, we inspected the ordering of the threshold parameters to examine the structure of the response scale. Thresholds should increase, indicating a move from low to high levels on a (self-efficacy) latent trait continuum. Reversed thresholds indicate that the response scale does not work as intended [29].

To study the targeting of the UW-SES-6, we examined the distributions of the person, item, and threshold parameters along the latent trait continuum. We also calculated the percentage of individuals with measures below the level of the lowest threshold, and of those with measures above the level of the highest threshold and analyzed the distance between the mean person location and the mean item location under consideration of the $95 \%$-confidence intervals [30].

Finally, we examined possible differential item functioning (DIF), or item bias, with respect to six subgroups: sex (male versus female), age (up to 48 versus 49 and above), education (finished high school, college or university versus low), level of lesion (para- versus tetraplegia), completeness of lesion (complete versus incomplete), and time since onset of injury (TSI) (up to 22 versus 23 or above). DIF indicates that individuals of different groups vary in their response probability to an item, although they share the same trait level [31]. We examined standardized residuals of all items using two-way analysis of variance with Bonferroni correction for multiple testing [32]. A significant main effect of the group (e.g., sex) or an interaction effect (e.g., sex $\times \mathrm{SE}$ ) is an indicator of DIF.

\section{Results}

A total of 282 individuals with SCI participated in the ALLRISC study, of whom 264 completed the questionnaire and 261 had complete UW-SES-6 data. Their demographic and SCI characteristics are displayed in Table 1. The distributions of the item scores are displayed in Table 2.

The results of the initial and final Rasch analyses are displayed in Table 3. Data from 13 participants were not used for this analysis because of extreme scores (0 or 100) on the UW-SES-6. The initial Rasch analysis revealed an acceptable fit. The Chi-square test showed misfit for item 1, but its $Z$ value was below 2.5. However, item 4 showed disordered thresholds. To solve this problem, we collapsed response options 3 and 4 in a second analysis. This solved the misfit problem, but the subsequent analysis of DIF showed DIF for sex in item 4. Collapsing response options 2 and 3 instead, in a third analysis, solved the DIF problem, but resulted in misfit of items 1 and 4 . Collapsing response

Table 1 Characteristics of the study sample $(N=261)$

\begin{tabular}{ll}
\hline Variable & \\
\hline Age (years), mean (SD); Md (IQR) & $48.5(8.8) ; 47.9$ (41.9-55) \\
TSI (years), mean (SD); Md (IQR) & $24.1(9.1) ; 22$ (16.8-30.3) \\
Gender (\% male) & 73.6 \\
Etiology of injury ${ }^{\mathrm{a}}(\%)$ & \\
$\quad$ Sports or leisure & 25.7 \\
$\quad$ Violence & 1.5 \\
$\quad$ Occupational & 10.7 \\
$\quad$ Road traffic accident & 45.2 \\
$\quad$ Fall & 9.2 \\
$\quad$ Non-traumatic SCI & 9.6 \\
Lesion level (\% tetraplegia) & 39.8 \\
Completeness lesion (\% motor & 81.6 \\
complete) & 96.2 \\
Nationality (\% Dutch) & 63.0 \\
Married or in stable relationship (\%) & 27.6 \\
Education (\% low) & 39.5 \\
Employment (\% paid employment)
\end{tabular}

Abbreviations: TSI time since injury, $M d$ median, and $I Q R$ interquartile range

${ }^{\text {a } M u l t i p l e ~ a n s w e r s ~ p o s s i b l e ~}$ 
Table 2 Distribution of scores on the short version of the University of Washington Self-Efficacy Scale (UW-SES-6) $(\mathrm{N}=261)$

Not at all A little Quite a bit A lot Completely

How confident are you that:

(1) You can keep the physical discomfort of your SCI from interfering with the things $13.4^{\mathrm{a}}$ you want to do

(2) You can keep your SCI from interfering with your ability to deal with unexpected 7.3 events
(3) You can keep your SCI from interfering with your ability to interact socially

\section{3}

(4) You can keep your SCI from being the center of your life

(5) You can bounce back from frustration, discouragement, or disappointment that SCI 3.4 may cause you

(6) You can figure out effective solutions to SCI-related issues that come up

$\begin{array}{rrrr}22.2 & 29.1 & 29.5 & 5.7 \\ 19.5 & 23.8 & 36.8 & 12.6 \\ & & & \\ 20.7 & 22.2 & 35.2 & 14.6 \\ 20.7 & 16.9 & 35.2 & 19.2 \\ 13.4 & 21.5 & 41.4 & 20.3 \\ & & & \\ 15.7 & 25.3 & 40.2 & 16.5\end{array}$

Abbreviations: $S C I$ spinal cord injury

${ }^{\mathrm{a}}$ Values are percentages

options 4 and 5 instead in the fourth analysis also solved the DIF problem, but still resulted in misfit of item 4 . Therefore we, finally, collapsed response options 2,3 , and 4 of item 4 . Table 3 shows that after this adjustment, the fifth and final Rasch analysis showed satisfactory item fit, with nonsignificant Chi-square values and all $Z$-values below 2.5. Local dependency was further absent, as shown by the $6.5 \%$ (95\% CI 4.1-9.6\%) significant $t$-tests between the subsets of items with positive versus negative factor loadings with the first residual factor, and that all residual correlations between item pairs were below 0.2 (mean across all residual correlations: -0.19 ).

The PSI of the UW-SES- 6 was 0.87 , indicating very good reliability. The mean differences between the locations of the thresholds were 2.69 between thresholds 1 and 2, 0.73 between thresholds 2 and 3, and 2.78 between thresholds 3 and 4 . This 0.73 is below the recommended range of differences of 1.4-5 logits [29]. This indicates that the differences between the response categories 2 and 3 are small.

The mean item location was 0 (by definition) with an SD of 0.64 (95\% CI -0.08 to 0.08 ). The mean person location was 0.60 (SD 1.95; 95\% CI 0.36-0.85). Analysis of possible DIF showed no item bias with respect to sex, age, education, level and completeness of lesion, and time since injury.

Figure 1 shows that the item thresholds are evenly distributed along the continuum of the latent trait (including the 13 participants with extreme values on the UW-SES-6). The person distribution shows a clustering between 0 and 2 logits above the mean.

Finally, a transformation table was compiled for the recoding of raw US-SES-6 scores into Rasch-based scores. This table is available from the first author.

\section{Discussion}

We studied the psychometric properties of the UW-SES-6 using Rasch-based methodology. After collapsing the three middle response options of one item, the UW-SES-6 was demonstrated to be a reliable unidimensional scale in people with long-standing SCI. Showing no item bias with respect to core demographic and SCI characteristics indicates that the UW-SES-6 can be used to compare scores between these subgroups.

The results of this study confirm and extend upon the positive psychometric properties reported in earlier studies [12, 18]. Amtmann et al. developed the UW-SES as an 17item scale and the UW-SES-6 as its short version, but did not publish psychometric data on this short version other than a high Cronbach's alpha (0.9) and the logit range ( -2.3 to 1$)$ in which the UW-SES-6 is sufficiently reliable to differentiate levels of $\mathrm{SE}$ at the individual level [12]. Further, Chung et al. showed equivalence of the UW-SES-6 scores across four diagnostic groups using structural equation modeling [18]. Our study, in addition, showed unidimensionality and measurement equivalence of this measure across subgroups of sex, age, level of education, time since onset of SCI, and type of SCI.

Our study further showed that the UW-SES-6 is welltargeted to the sample: the mean person location was only slightly higher than 0 and within $1 \mathrm{SD}$ of the mean item location, and only a low proportion (3.8\%) of participants showed the maximum score. This proportion is slightly lower compared to the $6 \%$ found by Amtmann et al. [12]. Despite collapsing three response categories of one item, the maximum range of the $T$-scores was with 18.7-76.1 slightly larger in our study compared to the maximum range of 20-68.9 found by Amtmann et al. [12].

Based on the results of our study, we advise people to administer the UW-SES-6 in its original form, but to collapse response categories 2, 3, and 4 of item 4 before using the transformation table (available from the corresponding author). People who want to report raw total scores can do the same, but recode the remaining three response categories as 1 , 3 , and 5. This way the scores of the lowest, middle, and the highest response categories, and thereby, the range of the raw 


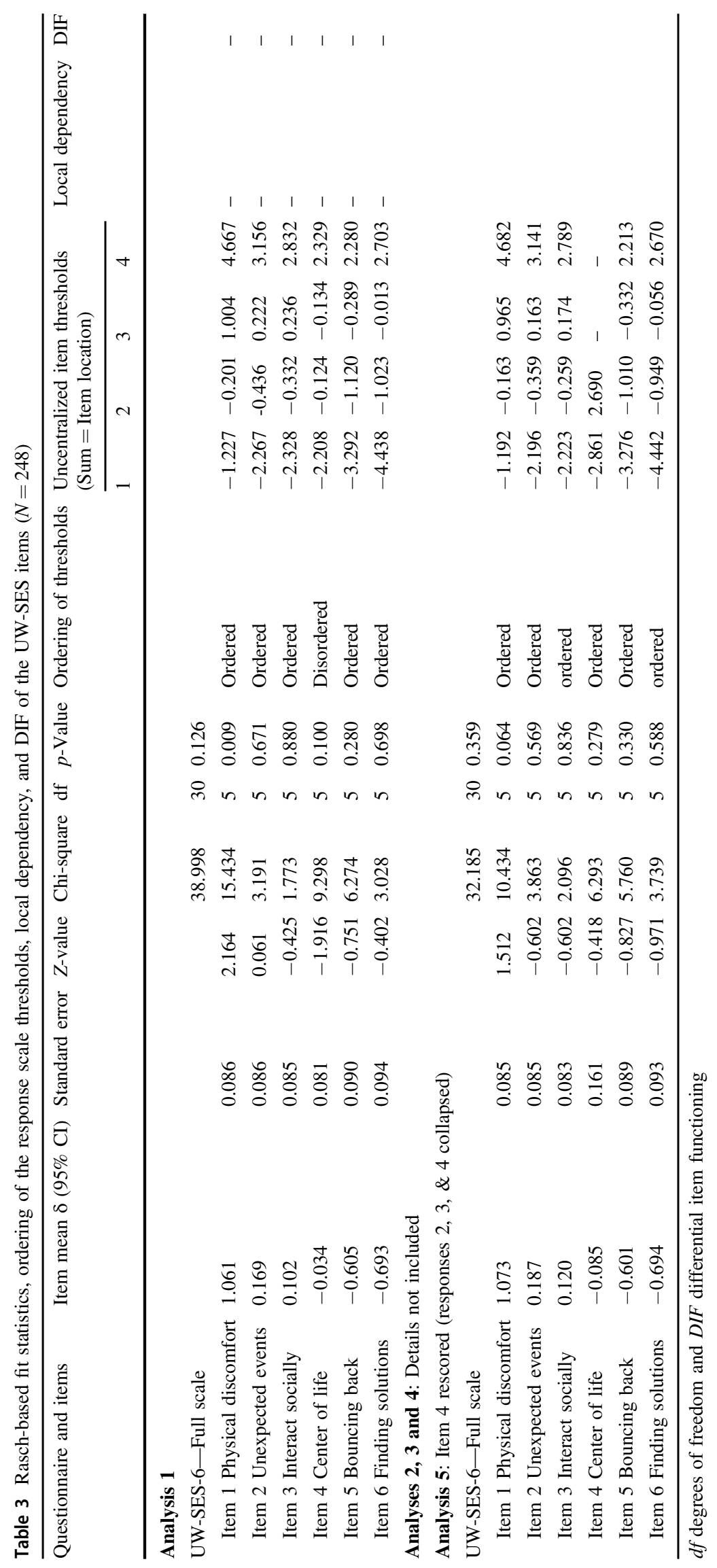




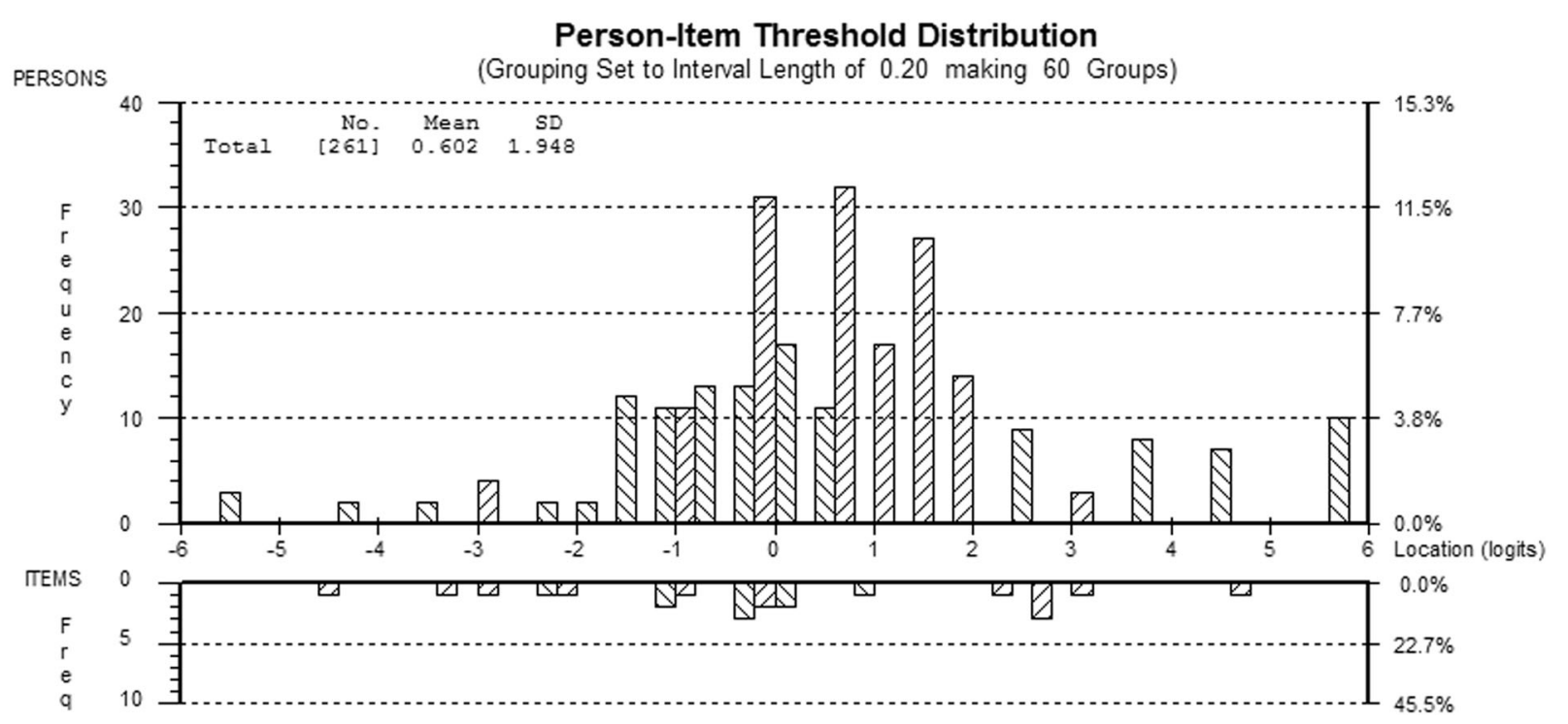

Fig. 1 Person and item distribution of the UW-SES-6 in people with long-standing SCI

total score (6-30) are retained. In our sample, more participants scored a 4 than a 2 on item 4 (Table 2). Therefore, this procedure resulted in slightly lower mean raw total score (19.9 compared to 20.0) and obviously a slightly lower standard deviation (5.2 instead of 5.5). Nevertheless, this procedure maximizes the comparability of item scores across items (compare with the thresholds in Table 3) and comparability with results from other studies.

\section{Limitations}

Due to the inclusion criteria of the ALLRISC study, the majority of our participants had a traumatic and a complete SCI and had acquired their SCI at a relatively young age. This may limit the generalization of our results to the whole population of people with SCI. Second, we used a Dutch translation of the UW-SES-6. Although the translation was carefully executed, we did not perform a back-translation and therefore the similarity of the Dutch and the American version needs further confirmation.

\section{Implications for further research}

The results of this study suggest that the American and Dutch versions may be more or less similar. Further research combining samples of different countries, however, is needed to study the psychometric equivalence of UW-SES-6 scores across countries/languages. Further research is also necessary to study the responsiveness of the UW-SES-6 in the context of intervention studies and to reveal how the UW-SES-6 relates to other measures of SE that have been used in people with SCI, such as the General SE Scale and the Moorong SE scale [16, 33].

\section{Clinical implications}

This study contributes to the psychometric evidence for the UW-SES-6 as a brief and valid instrument to measure DMSE among people with SCI. Since rehabilitation aims to teach people with recently acquired SCI to cope with the consequences of their condition in daily life, DMSE can be considered an important outcome of SCI rehabilitation. Also, SE has been associated with self-care behaviors, secondary conditions, and quality of life [9]. Therefore, it may be useful to use the UW-SES-6 to measure outcomes of rehabilitation and to screen for DMSE in individuals with SCI reporting ill-health or limitations arising from secondary health conditions, to identify those with low levels of DMSE and apply interventions to increase SE if appropriate [7].

\section{Conclusion}

This study showed the UW-SES-6 to be a scale with sound metric properties that has potential as a quick and easy selfreport measure for low SE in people with SCI.

Acknowledgments We thank all ALLRISC research assistants and SCI rehabilitation physicians for collecting all the data and the following participating Dutch rehabilitation centers: Rehabilitation Center De Hoogstraat (Utrecht), Reade Center for Rehabilitation (Amsterdam); Rehabilitation Center Het Roessingh (Enschede); Adelante Rehabilitation Center (Hoensbroek); Sint Maartenskliniek (Nijmegen); University Medical Center Groningen, Center for Rehabilitation-location Beatrixoord (Haren); Rehabilitation Center Heliomare (Wijk aan Zee), and Rijndam Rehabilitation Center (Rotterdam).

Author contributions MP was responsible for designing the study and writing the report, and contributed to the interpretation of the results. 
JA was responsible for the data collection and provided feedback on the report. CP was responsible for the statistical analyses and interpretation of the results, and provided feedback on this report.

Funding ALLRISC was sponsored by "Fonds NutsOHRA" under the responsibility of the Netherlands Organisation for Health Research and Development (www.ZonMW.nl), Project number 89000006.

\section{Compliance with ethical standards}

Conflict of interest The authors declare that they have no conflict of interest.

\section{References}

1. Adriaansen JJE, Post MWM, de Groot S, van Asbeck FWA, Stolwijk-Swüste JM, Tepper M, et al. Secondary health conditions in persons with spinal cord injury: a longitudinal study from one to five years post-discharge. J Rehabil Med. 2013;45:1016-22.

2. Noreau L, Noonan VK, Cobb J, Leblond J, Dumont FS. Spinal cord injury community survey: a national, comprehensive study to portray the lives of canadians with spinal cord injury. Top Spinal Cord Inj Rehabil. 2014;20:249-64.

3. Bloemen-Vrencken JHA, Post MWM, Hendriks JMS, de Reus ECE, de Witte LP. Health problems of persons with spinal cord injury living in the Netherlands. Disabil Rehabil. 2005;27:1381-9.

4. Brinkhof MWG, Al-Khodairy A, Eriks-Hoogland I, Fekete C, Hinrichs T, Hund-Georgiadis M, et al. Health conditions in people with spinal cord injury: contemporary evidence from a populationbased community survey in Switzerland. J Rehabil Med. 2016;48:197-209.

5. Post MW, van Leeuwen CM. Psychological issues in spinal cord injury: a review. Spinal Cord. 2012;50:382-9.

6. Van Leeuwen CMC, Kraaijeveld S, Lindeman E, Post MW. Associations between psychological factors and quality of life ratings in persons with spinal cord injury: a systematic review. Spinal Cord. 2012;50:174-87.

7. Peter C, Muller R, Cieza A, Geyh S. Psychological resources in spinal cord injury: a systematic literature review. Spinal Cord. 2012;5:188-201.

8. Bandura A. Self-efficacy: toward a unifying theory of behavioral change. Psychol Rev. 1977;84:191-215.

9. Van Diemen T, Crul T, van Nes I, SELF-SCI Group, Geertzen JHB, Post MWM. Associations between self-efficacy and secondary health conditions in patients living with spinal cord injury: a systematic review and meta-analysis. Arch Phys Med Rehabil. 2017;98:2566-77.

10. Sherer M, Maddux JE, Mercandante B, Prentice-Dunn S, Jacobs B, Rogers RW. The self-efficacy scale: construction and validation. Psychol Rep. 1982;51:663-71.

11. Lorig KR, Sobel DS, Ritter PL, Laurent D, Hobbs M. Effect of a self-management program on patients with chronic disease. Eff Clin Pract. 2001;4:256-62.

12. Amtmann D, Bamer AM, Cook KF, Askew RL, Noonan VK, Brockway JA. University of Washington Self-Efficacy Scale: a new self-efficacy scale for people with disabilities. Arch Phys Med Rehabil. 2012;93:1757-65.

13. Fliess-Douer O, van der Woude LH, Vanlandewijck YC. Development of a new scale for perceived self-efficacy in manual wheeled mobility: a pilot study. J Rehabil Med. 2011;43:602-8.
14. King RB, Champion VL, Chen D, Gittler MS, Heinemann AW, Bode RK, et al. Development of a measure of skin care belief scales for persons with spinal cord injury. Arch Phys Med Rehabil. 2012;93:1814-21.

15. van Diemen T, Scholten E, van Nes I, Geertzen J, Post M. Longitudinal cohort study on self-management and self-efficacy in Dutch initial spinal cord injury rehabilitation: study protocol of the SELFSCI study. JMIR Res Protoc. 2018;7:e68.

16. Middleton JW, Tate RL, Geraghty TJ. Self-efficacy and spinal cord injury: psychometric properties of a new scale. Rehabil Psychol. 2003;48:281-8.

17. Middleton JW, Tran Y, Lo C, Craig A. Reexamining the validity and dimensionality of the Moorong Self-Efficacy Scale: improving its clinical utility. Arch Phys Med Rehabil. 2016;97:2130-6.

18. Chung H, Kim J, Park R, Bamer AM, Bocell FD, Amtmann D. Testing the measurement invariance of the University of Washington Self-Efficacy Scale short form across four diagnostic subgroups. Qual Life Res. 2016;25:2559-64.

19. Cijsouw A,Adriaansen JJ,Tepper M,Dijksta CA,van Linden S, ALLRISC . et al. Associations between disability-management self-efficacy, participation and life satisfaction in people with long-standing spinal cord injury. Spinal Cord. 2017;55:47-51.

20. Wright BD, Linacre JM. Observations are always ordinal; measurements, however, must be interval. Arch Phys Med Rehabil. 1989;70:857-60.

21. Adriaansen JJE, van Asbeck FWA, Lindeman E, van der Woude LHV, de Groot S, Post MWM. Secondary health conditions in persons with a spinal cord injury for at least 10 years: design of a comprehensive long-term cross-sectional study. Disabil Rehabil. 2013;35:1104-10.

22. Kirshblum SC, Burns SP, Biering-Sorensen F, DonovanW, Graves DE, Jha A, et al. International standards for neurological classification of spinal cord injury (revised 2011). J Spinal Cord Med. 2011;34:535-46.

23. Andrich D, Sheridan B, Luo G. Rasch models for measurement: RUMM2030. Perth, Western Australia: RUMM Laboratory Pty Ltd; 2010.

24. Tennant A, Conaghan PG. The Rasch measurement model in rheumatology: what is it and why use it? When should it be applied, and what should one look for in a Rasch paper? Arthritis Rheum. 2007;57:1358-62.

25. Andrich D. Rasch models for measurement. Newbury Park, CA: Sage; 1988.

26. Smith EV Jr. Detecting and evaluating the impact of multidimensionality using item fit statistics and principal component analysis of residuals. J Appl Meas. 2002;3:205-31.

27. Lundgren-Nilsson $\AA$, Tennant A. Past and present issues in Rasch analysis: the Functional Independence Measure $\left(\right.$ FIM $\left.^{\mathrm{TM}}\right)$ revisited. J Rehabil Med. 2011;43:884-91.

28. Nunally JC, Bernstein IH. Psychometric theory. 3rd ed.. New York: McGraw-Hill; 1994.

29. Linacre JM. Optimizing rating scale category effectiveness. J Appl Meas. 2002;2001:85-106.

30. Bond TG, Fox CM. Applying the Rasch model: fundamental measurement in the human sciences. Mahwah, NJ: Lawrence Erlbaum Associates; 2001.

31. Teresi J, Fleishman J. Differential item functioning and health assessment. Qual Life Res. 2007;16:33-42.

32. Bland JM, Altman DG. Multiple significance tests: the Bonferroni method. Br Med J. 1995;310:170.

33. Peter C, Cieza A, Geyh S. Rasch analysis of the General SelfEfficacy Scale in spinal cord injury. J Health Psychol. 2014;19:544-55. 